Gut and Liver, Vol. 10, No. 1, January 2016, pp. 69-75

\title{
Does Helicobacter pylori Exacerbate Gastric Mucosal Injury in Users of Nonsteroidal Anti-Inflammatory Drugs? A Multicenter, Retrospective, Case-Control Study
}

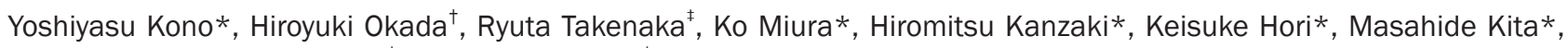
Takao Tsuzuki*, Seiji Kawano ${ }^{\dagger}$, Yoshiro Kawahara ${ }^{\dagger}$, and Kazuhide Yamamoto*

*Department of Gastroenterology and Hepatology, Okayama University Graduate School of Medicine, Dentistry and Pharmaceutical Sciences, ${ }^{\dagger}$ Department of Endoscopy, Okayama University Hospital, and ${ }^{\ddagger}$ Department of Internal Medicine, Tsuyama Chuo Hospital, Okayama, Japan

Background/Aims: The interaction between nonsteroidal anti-inflammatory drugs (NSAIDs) and Helicobacter pylori remains controversial. We retrospectively investigated whether H. pylori infection exacerbates severe gastric mucosal injury among chronic NSAID users. Methods: From January 2010 to December 2013, a total of 245 long-term NSAID (including low-dose aspirin) users who had undergone an esophagogastroduodenoscopy and had been evaluated for $\mathrm{H}$. pylori infection were enrolled at Okayama University Hospital and Tsuyama Chuo Hospital. The degree of gastric mucosal injury was assessed according to the modified Lanza score (MLS). Severe gastric mucosal injury was defined as an MLS $\geq 4$. Univariate and multivariate logistic regression analyses were performed. Results: In the univariate analysis, age $\geq 75$ years (odds ratio [OR], 2.4; 95\% confidence interval [Cl], 1.3 to 4.2), H. pylori-positivity (OR, 2.0; $95 \% \mathrm{Cl}, 1.2$ to 3.5), and the concomitant use of proton pump inhibitors (PPIs) (OR, 0.48; $95 \% \mathrm{Cl}, 0.26$ to 0.86 ) were significantly associated with severe gastric mucosal injury. The multivariate analysis was adjusted by age and sex and demonstrated that $H$. pyloripositivity (OR, 1.8; 95\% $\mathrm{Cl}, 1.0$ to 3.3) and the concomitant use of PPIs (OR, 0.53; 95\% Cl, 0.28 to 0.99) significantly contributed to severe gastric mucosal injury. Conclusions: H. pylori infection exacerbates severe gastric mucosal injury among chronic NSAID users. (Gut Liver 2016;10:69-75)

Key Words: Anti-inflammatory agents, non-steroidal; Helicobacter pylori; Gastric mucosal injury; Proton pump inhibitors

\section{INTRODUCTION}

The use of nonsteroidal anti-inflammatory drugs (NSAIDs) including low-dose aspirin (LDA) has increased in recent years, and their use is known to increase the risk of gastric mucosal injury and ulcers. ${ }^{1,2}$ It has been reported that the risk factors for future NSAIDs-associated ulcer complications include a history of uncomplicated peptic ulcer, advanced age, comorbidities, high-dose use, and use of concomitant drugs such as antiplatelet drugs and anticoagulants.,

Gastric acid is another important factor involved in NSAIDsinduced gastric mucosal injury, ${ }^{5,6}$ and Helicobacter pylori infection is known to exert diverse effects on gastric acid secretion; infection can cause an increase, decrease, or no change in gastric acid secretion depending on the distribution of inflammation or presence of atrophy within the stomach. ${ }^{7}$ Thus, the interaction between $H$. pylori and NSAIDs may vary, according to the topography and severity of gastritis. In 2012, Iijima and colleagues ${ }^{8}$ reported that the effect of $H$. pylori infection on aspirin-induced gastropathy was biphasic depending on the patient's gastric acid secretion level. The interaction between NSAIDs and $H$. pylori remains incompletely understood and controversial.

There is a paucity of data regarding the interaction between NSAIDs-induced gastric mucosal injury and $H$. pylori infection in Japanese patients because almost all studies to date have been conducted in Western countries. Moreover, the incidence of NSAIDs-induced gastric mucosal injury in $H$. pylori-positive patients has not been adequately studied although the endpoint in most studies was the incidence of peptic ulcer or bleeding in

\footnotetext{
Correspondence to: Yoshiyasu Kono

Department of Gastroenterology and Hepatology, Okayama University Graduate School of Medicine, Dentistry and Pharmaceutical Sciences, 2-5-1 Shikata-cho, Kita-ku, Okayama 700-8558, Japan

Tel: +81-86-235-7219, Fax: +81-86-225-5991, E-mail: hxnwq178@yahoo.co.jp

Received on September 25, 2014. Revised on October 25, 2014. Accepted on December 9, 2014. Published online June 19, 2015

pISSN 1976-2283 eISSN 2005-1212 http://dx.doi.org/10.5009/gnl14372

@) This is an Open Access article distributed under the terms of the Creative Commons Attribution Non-Commercial License (http://creativecommons.org/licenses/by-nc/4.0) which permits unrestricted non-commercial use, distribution, and reproduction in any medium, provided the original work is properly cited.
} 
NSAIDs users. ${ }^{1,9,10}$

The aim of this study was to assess whether $H$. pylori infection exacerbates severe gastric mucosal injury associated with chronic NSAIDs use.

\section{MATERIALS AND METHODS}

\section{Study design and subjects}

This was a multicenter, retrospective, case-control study. From January 2010 to December 2013, a total of 245 consecutive outpatients and inpatients who had been taking a nonaspirin NSAID (NANSAID) or LDA (75 to $325 \mathrm{mg}$ ) continuously for at least 3 months and had undergone esophagogastroduodenoscopy were retrospectively enrolled at Okayama University Hospital and Tsuyama Chuo Hospital. Exclusion criteria for the participants included a history of endoscopic resection or gastric surgery for gastric cancer; gastric/duodenal ulcer, or $H$. pylori eradication treatment; serious systemic diseases; presence of gastric cancer; and age $<20$ years. Surveyed background factors were age, gender, degree of gastric mucosal injury, degree of gastric atrophy, incidence of $H$. pylori infection, type of NSAIDs used (NANSAID alone, LDA alone, or both NANSAID and LDA), and concomitant medications (antiplatelet agents, anticoagulants, or antisecretory agents). The study was approved by the Okayama University School of Medicine and Tsuyama Chuo Hospital Clinical Ethics Committee on Human Experiments, in accordance with the Helsinki Declaration, and each subject gave their written informed consent.

\section{Endoscopic evaluation of gastric mucosal injury and gastric atrophy}

The degree of gastric mucosal injury was assessed according to the modified Lanza score (MLS). ${ }^{11}$ In this scoring system, gastric mucosal injury is graded according to six categories on a scale from 0 to 5: grade 0 , no erosion/hemorrhage; grade 1, 1-2 lesions of erosion and/or hemorrhage localized in one area of the stomach; grade 2, 3-5 lesions of erosion and/or hemorrhage localized in one area of the stomach; grade 3, 6-9 lesions of erosion and/or hemorrhage localized in one area of the stomach, or no more than 10 lesions in two areas of the stomach; grade 4 , erosions and/or hemorrhage in three areas of the stomach, or no fewer than 10 lesions in the whole stomach; and grade 5, a gastric ulcer, defined as a mucosal defect larger than $5 \mathrm{~mm}$ in diameter.

The degree of atrophy was diagnosed according to the criteria of Kimura and Takemoto ${ }^{12}$ as reported previously. The MLS and degree of endoscopic atrophy for all subjects were graded independently in a blinded manner by two endoscopists. When there was disagreement, a consensus was reached through joint review of the endoscopic pictures. In the present analysis, severe gastric mucosal injury was defined as an MLS $\geq 4$.

\section{Diagnosis of H. pylori infection}

All subjects were evaluated for $H$. pylori infection. Histology, H. pylori IgG antibody, rapid urease test (RUT), or urea breath test were used to detect $H$. pylori infection. $H$. pylori infection was diagnosed when any of the studies was positive. For histology, three biopsy specimens (greater curvature of antrum, lesser curvature of lower gastric body, and greater curvature of upper body) were fixed with buffered formalin and embedded in paraffin. Sections were stained with Giemsa stain solution. An expert pathologist from each of the two centers who was unaware of the endoscopic findings assessed $H$. pylori infection and graded the degree of histological gastritis in each biopsy sample. ${ }^{14}$ For H. pylori IgG antibody, we studied the titers of serum or urinary IgG antibodies against $H$. pylori, using an enzyme immunoassay (EIA kit) (E Plate "Eiken" H. pylori-antibody; Eiken Chemical, Tokyo, Japan), ${ }^{15}$ or RAPIRUN H. pylori antibody detection kit (Otsuka Pharmaceutical Co., Ltd., Tokyo, Japan). ${ }^{16}$ The two gastric biopsies taken for RUT were immediately dropped into Helicocheck $^{\mathrm{TM}}$ (Otsuka Pharmaceutical Co., Ltd., Osaka, Japan). $H$. pylori was identified on the basis of a color change from yellow to red after 2 hours. ${ }^{13} \mathrm{C}$-urea breath testing (UbiT; Otsuka Pharmaceuticals, Tokyo, Japan) was also used. The cutoff value for $\Delta^{13} \mathrm{CO}_{2}$ was set at $2.5 \%{ }^{17}$

\section{Statistical analysis}

Mann-Whitney U test was used for continuous variables, and Fisher exact test or chi-square test were used for categorical variables between $H$. pylori-negative and $H$. pylori-positive subjects.

To identify significant factors contributing to NSAIDs-induced severe gastric mucosal injury, univariate and multivariate logistic regression analyses were performed. Variables found in the univariate analysis to be significantly associated were included in a multivariate logistic regression analysis. Valuables such as age and gender were also included in the multivariate regression analysis to adjust for the effect of confounding factors. The results of analysis were expressed as odds ratio (OR) and confidence interval (CI). All statistical calculations were carried out using JMP software (for Windows, version 10). The $\mathrm{p}$ values $<0.05$ were considered to be statistically significant in all tests.

\section{RESULTS}

\section{Clinical parameters in all subjects}

Clinical parameters in all subjects are shown in Table 1. About one-third (32\%) had severe gastric mucosal injury, and the incidence of $H$. pylori infection and open-type gastric atrophy were $44 \%$ and $43 \%$, respectively. Roughly 50\% of subjects concomitantly used antisecretory drugs such as proton pump inhibitors (PPIs) or histamine $\mathrm{H}_{2}$-receptor antagonists $\left(\mathrm{H}_{2}-\mathrm{RAs}\right)$. 
Table 1. Clinical Parameters of the 245 Cases

\begin{tabular}{lc}
\hline \multicolumn{1}{c}{ Parameter } & Value \\
\hline Age, yr & $65 \pm 14$ \\
Gender, female/male & $120 / 125$ \\
MLS & $1.9 \pm 2.2$ \\
MLS $\geq 4$ & $79(32.2)$ \\
Degree of gastric atrophy & \\
None or closed & $140(57.1)$ \\
Open & $105(42.9)$ \\
H. pylori infection & $137(55.9)$ \\
Negative & $108(44.1)$ \\
Positive & \\
Type of NSAIDs & $119(48.6)$ \\
NANSAID alone & $114(46.5)$ \\
LDA alone & $12(4.9)$ \\
Both NANSAID and LDA & \\
Concomitant medications & $49(20.0)$ \\
Antiplatelet agents & $18(7.3)$ \\
Anticoagulants & $127(51.8)$ \\
Antisecretary agents & $85(34.7)$ \\
PPIs & $42(17.1)$ \\
$\quad \mathrm{H}_{2}-$ RAs &
\end{tabular}

Data are presented as mean \pm SD or number $(\%)$.

MLS, modified Lanza score; H. pylori, Helicobacter pylori; NSAID, nonsteroidal anti-inflammatory drugs; NANSAID, nonaspirin NSAID; LDA, low-dose aspirin; PPI, proton pump inhibitor; $\mathrm{H}_{2}-\mathrm{RA}$, histamine $\mathrm{H}_{2}$-receptor antagonist.

Table 2. Comparison of the Clinical Parameters between $H$. pyloriNegative and $H$. pylori-Positive Subjects

\begin{tabular}{lccc}
\hline \multicolumn{1}{c}{ Parameter } & $\begin{array}{c}\text { H. pylori- } \\
\text { negative } \\
(\mathrm{n}=137)\end{array}$ & $\begin{array}{c}\text { H. pylori- } \\
\text { positive } \\
(\mathrm{n}=108)\end{array}$ & p-value \\
\hline Age, $\geq 75$ yr & $41(29.9)$ & $33(30.6)$ & 1.0 \\
Gender, female/male & $67 / 70$ & $53 / 55$ & 1.0 \\
Type of NSAIDs & $70(51.1)$ & $49(45.4)$ & 0.25 \\
$\quad$ NANSAID alone & $59(43.1)$ & $55(50.9)$ & 0.44 \\
LDA alone & $8(5.8)$ & $4(3.7)$ & 0.56 \\
$\quad$ Both NANSAID and LDA & & & \\
Concomitant medications & $27(19.7)$ & $22(20.4)$ & 1.0 \\
$\quad$ Antipletelet agents & $7(5.1)$ & $11(10.2)$ & 0.15 \\
Anticoagulants & $83(60.6)$ & $44(40.7)$ & 0.003 \\
Antisecretary agents & $61(44.5)$ & $24(22.2)$ & $<0.001$ \\
$\quad$ PPIs & $22(16.1)$ & $20(18.5)$ & 0.61 \\
$\quad H_{2}$-RAs & $39(28.5)$ & $66(61.1)$ & $<0.001$ \\
\hline Open-type gastric atrophy & & & \\
\hline
\end{tabular}

Data are presented as mean \pm SD or number $(\%)$.

H. pylori, Helicobacter pylori; NSAID, nonsteroidal anti-inflammatory drug; NANSAID, nonaspirin NSAID; LDA, low-dose aspirin; PPI, proton pump inhibitor; $\mathrm{H}_{2}-\mathrm{RA}$, histamine $\mathrm{H}_{2}$-receptor antagonist.

\section{Comparison of the degree of NSAIDs-induced gastric mucosal injury between $\boldsymbol{H}$. pylori-negative and $\boldsymbol{H}$. pylori- positive subjects}

Clinical parameters in $H$. pylori-negative and $H$. pylori-positive subjects are shown in Table 2. No significant differences were seen in terms of age, gender, type of NSAIDs used, or concomitant medications (antiplatelet agents and anticoagulants). The percentage of open-type gastric atrophy was significantly higher ( $61 \%$ vs $28 \%, \mathrm{p}<0.001$ ), and concomitant use of PPIs was significantly lower (22\% vs $45 \%, \mathrm{p}<0.001$ ) in $H$. pylori-positive subjects than in $H$. pylori-negative subjects, respectively.

As shown in Figs 1 and 2, the mean MLS (2.3 vs 1.7, p=0.037) and the percentage of patients with an MLS of $\geq 4$ ( $41 \%$ vs $26 \%$, $\mathrm{p}=0.013$ ) were both higher in $H$. pylori-positive subjects than in $H$. pylori-negative subjects. Moreover, we investigated the rela-

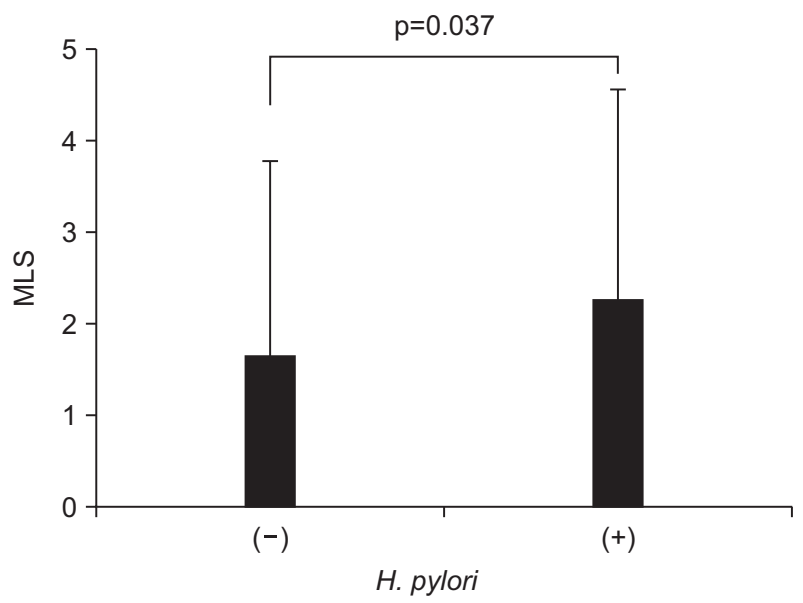

Fig. 1. Comparison of the modified Lanza score (MLS) between Helicobacter pylori-negative subjects and $H$. pylori-positive subjects in all cases. The mean MLS was significantly higher in $H$. pylori-positive subjects than in $H$. pylori-negative subjects (each column indicates the mean $\pm \mathrm{SD} ; 2.3 \pm 2.3$ vs $1.7 \pm 2.1, \mathrm{p}=0.037$ ).

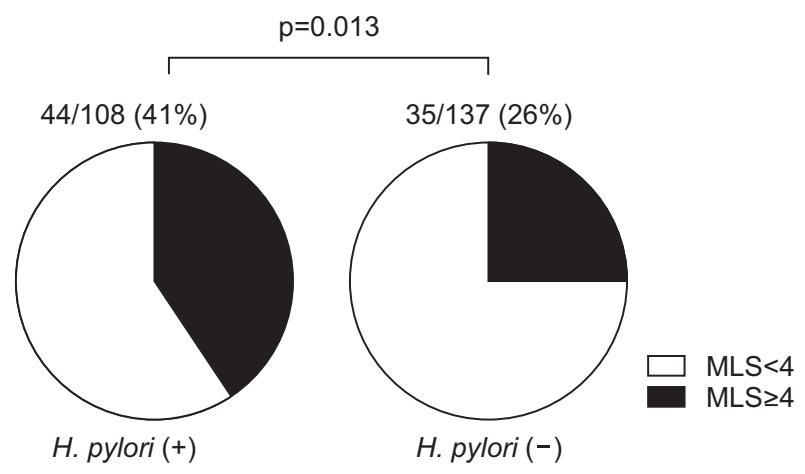

Fig. 2. Comparison of the incidence of severe gastric mucosal injury (modified Lanza score $[\mathrm{MLS}] \geq 4$ ) between Helicobacter pylorinegative subjects and $H$. pylori-positive subjects in all cases. The percentage of patients with MLS $\geq 4$ was significantly higher among $H$. pylori-positive patients than in $H$. pylori-negative patients ( $41 \%$ vs $26 \%, p=0.013)$. 
tionship between degree of gastric atrophy with $H$. pylori infection and MLS (Figs 3 and 4). Among the subjects with none or closed-type gastric atrophy, the mean MLS was higher (2.3 vs 1.6, $\mathrm{p}=0.093$ ), and the percentage of patients with MLS $\geq 4$ was significantly higher ( $43 \%$ vs $24 \%, \mathrm{p}=0.043$ ) in $\mathrm{H}$. pylori-positive subjects than in $H$. pylori-negative subjects. Among the subjects with open-type gastric atrophy, however, the mean MLS and the percentage of patients with MLS $\geq 4$ was not significantly higher in $H$. pylori-positive subjects than $H$. pylori-negative subjects $(2.2$ vs $1.7, p=0.28$, and $39 \%$ vs $28 \%, p=0.29$, respectively).

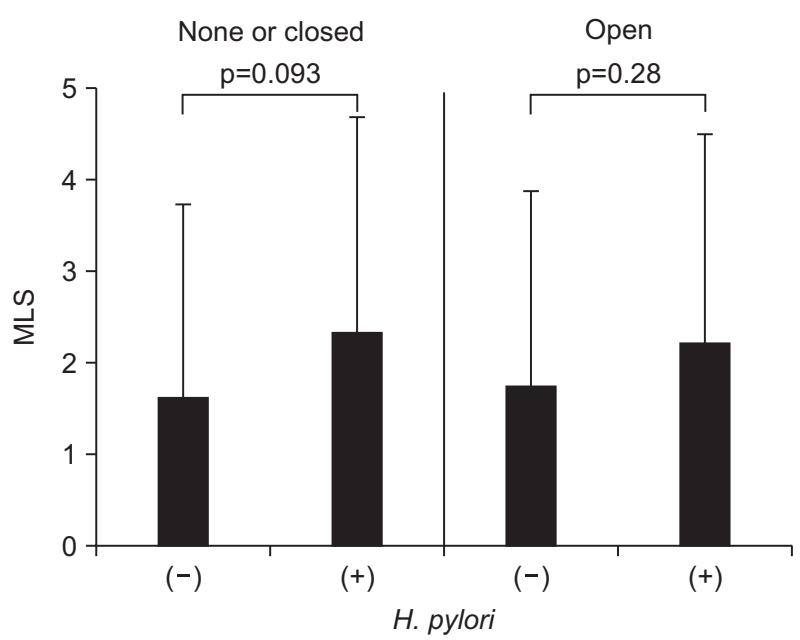

Fig. 3. Comparison of the modified Lanza score (MLS) between Helicobacter pylori-negative subjects and $H$. pylori-positive subjects, according to the degree of gastric atrophy. The difference in the mean MLS between $H$. pylori-negative and $H$. pylori-positive subjects was larger among the subjects with no or closed-type gastric atrophy than among those with open-type gastric atrophy (each column indicates the mean $\pm \mathrm{SD} ; 1.6 \pm 2.1$ vs $2.3 \pm 2.4, \mathrm{p}=0.093$ in the subjects with no or closed-type gastric atrophy, and $1.7 \pm 2.2$ vs $2.2 \pm 2.3, \mathrm{p}=0.28$ in the subjects with open-type gastric atrophy, respectively).

\section{Factors contributing to NSAIDs-induced severe gastric mucosal injury}

Table 3 shows the results of logistic regression analysis for factors contributing to NSAIDs-induced severe gastric mucosal injury (MLS $\geq 4$ ). In univariate analysis, age $\geq 75$ years (OR, 2.4; 95\% CI, 1.3 to 4.2 ), $H$. pylori-positivity (OR, 2.0; 95\% CI, 1.2 to 3.5), and concomitant use of PPIs (OR, 0.48; 95\% CI, 0.26 to $0.86)$ were significantly related to NSAIDs-induced severe gastric mucosal injury. In multivariate analysis adjusted by age and gender, $H$. pylori-positivity (OR, 1.8; 95\% CI, 1.0 to 3.3), and concomitant use of PPIs (OR, 0.53; 95\% CI, 0.28 to 0.99) were significant factors associated with NSAIDs-induced severe gastric mucosal injury.

\section{DISCUSSION}

In summary, we investigated the effect of $H$. pylori infection on severe gastric mucosal injury among chronic NSAIDs users. The incidence of NSAIDs-induced severe gastric mucosal injury was higher in $H$. pylori-positive subjects than in $H$. pylori-negative subjects, especially among the subjects with none or closed type gastric atrophy. In multivariate regression analysis adjusted by age and gender, $H$. pylori was identified as a significantly aggressive factor contributing to NSAIDs-induced severe gastric mucosal injury.

Regarding the interaction between $H$. pylori and NSAIDs on gastric mucosal injury, several contributing factors are possibly working. First, $H$. pylori affects gastric acid secretion depending on the severity and phenotype of the induced gastritis. ${ }^{10,18}$ It has been reported that $H$. pylori infection has no significant effect on aspirin-induced mucosal injury after adjusting for gastric acid secretion. ${ }^{19}$ Alternatively, we investigated the relationship between degree of gastric atrophy with $H$. pylori infection and MLS, because gastric atrophy, which is closely related to $H$.

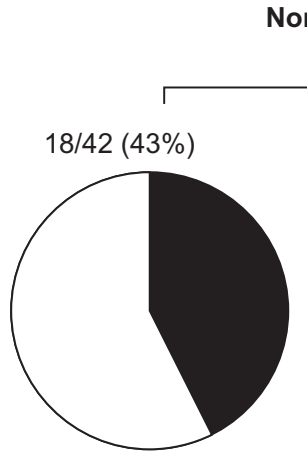

H. pylori (+)

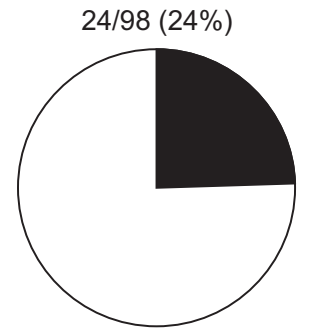

H. pylori (-)

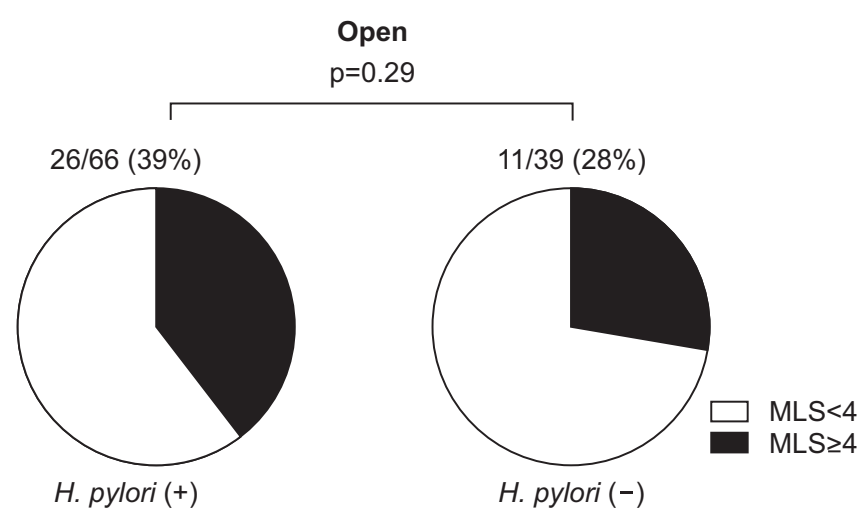

Fig. 4. Comparison of the incidence of severe gastric mucosal injury (modified Lanza score [MLS] $\geq 4$ ) between Helicobacter pylori-negative and H. pylori-positive subjects, according to the degree of gastric atrophy. Among the subjects with no or closed-type gastric atrophy, the percentage of patients with MLS $\geq 4$ was significantly higher in $H$. pylori-positive than in $H$. pylori-negative subjects; however, it was not significantly higher among the subjects with open-type gastric atrophy (43\% vs $24 \%, \mathrm{p}=0.043$ in the subjects with no or closed-type gastric atrophy, and $39 \%$ vs $28 \%, p=0.29$ in the subjects with open-type gastric atrophy, respectively). 
Table 3. Univariate and Multivariate Logistic Regression Analysis of Factors Contributing to Nonsteroidal Anti-Inflammatory Drugs-Induced Severe Gastric Mucosal Injury (Modified Lanza Score $\geq 4$ )

\begin{tabular}{|c|c|c|c|}
\hline Factor & Subgroup & Univariate OR $(95 \% \mathrm{CI})$ & Multivariate OR $(95 \% \mathrm{CI})$ \\
\hline \multirow[t]{2}{*}{ Age } & $<75 \mathrm{yr}$ & 1.0 & 1.0 \\
\hline & $\geq 75 \mathrm{yr}$ & $2.4(1.3-4.2)$ & $2.6(1.4-4.7)^{\dagger}$ \\
\hline \multirow[t]{2}{*}{ Gender } & Female & 1.0 & 1.0 \\
\hline & Male & $1.5(0.90-2.7)$ & $1.6(0.92-2.9)$ \\
\hline \multicolumn{4}{|l|}{ Type of NSAIDs } \\
\hline \multirow[t]{2}{*}{ NANSAID/LDA } & NANSAID & 1.0 & - \\
\hline & LDA & $1.0(0.56-1.6)$ & - \\
\hline \multirow[t]{2}{*}{ Both NANSAID and LDA } & No & 1.0 & - \\
\hline & Yes & $1.1(0.27-3.5)$ & - \\
\hline \multicolumn{4}{|l|}{ Concomitant medications } \\
\hline \multirow[t]{2}{*}{ Antiplatelet agents } & No & 1.0 & - \\
\hline & Yes & $0.91(0.45-1.8)$ & - \\
\hline \multirow[t]{2}{*}{ Anticoagulants } & No & 1.0 & - \\
\hline & Yes & $0.58(0.16-1.7)$ & - \\
\hline \multicolumn{4}{|l|}{ Antisecretary agents } \\
\hline \multirow[t]{2}{*}{ PPIs } & No & 1.0 & 1.0 \\
\hline & Yes & $0.48(0.26-0.86)^{*}$ & $0.53(0.28-0.99)^{*}$ \\
\hline \multirow[t]{2}{*}{$\mathrm{H}_{2}-\mathrm{RAs}$} & No & 1.0 & - \\
\hline & Yes & $0.61(0.27-1.3)$ & - \\
\hline \multirow[t]{2}{*}{ Degree of gastric atrophy } & None or closed & 1.0 & - \\
\hline & Open & $1.3(0.74-2.2)$ & - \\
\hline \multirow[t]{2}{*}{ H. pylori infection } & Negative & 1.0 & 1.0 \\
\hline & Positive & $2.0(1.2-3.5)^{*}$ & $1.8(1.0-3.3)^{\dagger}$ \\
\hline
\end{tabular}

OR, odds ratio; CI, confidence interval; NSAID, nonsteroidal anti-inflammatory drugs; NANSAID, nonaspirin NSAID; LDA, low-dose aspirin; PPI, proton pump inhibitor; $\mathrm{H}_{2}-\mathrm{RA}$, histamine $\mathrm{H}_{2}$-receptor antagonist; $H$. pylori, Helicobacter pylori.

${ }^{*} \mathrm{p}<0.05 ;{ }^{\dagger} \mathrm{p}<0.01$.

pylori infection, is associated with a decline in gastric acid secretion. ${ }^{20}$ As shown in Figs 3 and 4, H. pylori infection exacerbated NSAIDs-induced gastric mucosal injury among those with open-type gastric atrophy, but it was not statistically significant. In larger study, we may be able to investigate that $H$. pylori infection has a synergic effect on NSAID-induced gastric mucosal injury among those with open-type gastric atrophy, as similarly as among those with none or closed-type gastric atrophy. However, we confirmed in this study that $H$. pylori infection had more synergic effect on NSAIDs-induced gastric mucosal injury among the subjects with none or closed-type gastric atrophy than among those with open-type gastric atrophy. In addition, the different phenotypes of $H$. pylori gastritis may contribute to the contradictory data concerning its effect on gastric mucosal injury in NSAIDs users. In Western countries, several studies have demonstrated that $H$. pylori infection and NSAIDs use represent synergistic risk factors for uncomplicated and bleeding peptic ulcer. ${ }^{1,21}$ Furthermore, $H$. pylori exacerbated NSAIDsinduced gastric mucosal injury in our study. These results may be not only due to the degree of gastric acid secretion as men- tioned before, but due to a substantial inflammatory reaction in the gastric mucosa with recruitment of leukocytes and overexpression and release of proinflammatory cytokines induced by H. pylori. ${ }^{18,22}$ The phenomenon of reduced gastric mucosal injury despite repeated doses of NSAIDs (termed adaptation) also should be considered. ${ }^{23} \mathrm{H}$. pylori is thought to impair adaptation to NSAIDs-induced gastric mucosal injury. ${ }^{24}$ The subjects in this study were long-term NSAIDs users, so this impairment of adaptation by $H$. pylori may have also contributed to our results.

Based on our findings, concomitant use of PPIs, but not $\mathrm{H}_{2}$ RAs, can reduce the risk of NSAIDs-induced severe gastric mucosal injury. Several studies have fully examined the efficacy of PPIs and high-dose $\mathrm{H}_{2}$-RAs for preventing NSAIDs-induced erosion/ulcer. ${ }^{25-27}$ In our study, normal-dose $\mathrm{H}_{2}$-RAs users were also enrolled, and the number of concomitant $\mathrm{H}_{2}$-RAs users was small. This may explain why concomitant use of $\mathrm{H}_{2}$-RAs was not a significant preventive factor for NSAIDs-induced severe gastric mucosal injury in this study.

As shown in Table 2, the percentage of concomitant use of PPI was significantly lower in $H$. pylori-positive patients than in 
H. pylori-negative patients. Possible explanations for this findings are as follows: the gastric acid levels in $H$. pylori-positive patients could be lower because the incidence of open-type gastric atrophy was higher in $H$. pylori-positive patients than in $H$. pylori-negative patients. The higher gastric acid levels could contribute to the digestive symptoms such as heartburn, chest pain, or epigastralgia. That was why the $H$. pylori-negative patients used PPI more frequently than $H$. pylori-positive patients. Moreover, we could suggest that PPI was effective for chronic NSAIDs-users in $H$. pylori-positive patients even if they had less gastric acid levels, because the concomitant PPI users in $H$. pylori-positive patients had less injured gastric mucosa status in our study (Supplementary Fig. 1).

Regarding the secondary prevention of peptic ulcer or bleeding, it has been reported that $H$. pylori eradication has no beneficial effect compared with placebo in chronic NSAIDs users, ${ }^{28,29}$ and PPIs were more effective than $H$. pylori eradication. ${ }^{30,31}$ On the other hand, for primary prevention in chronic NSAIDs users, there is limited evidence indicating the effectiveness of $H$. pylori eradication. ${ }^{32}$ The reduced gastric acid secretion would be normalized as a result of eradication of $\mathrm{H}$. pylori, ${ }^{33}$ so that gastric mucosal injury in chronic NSAIDs users may be exacerbated after $H$. pylori eradication. It is therefore important to investigate changes in gastric mucosal injury among chronic NSAIDs users before and after $H$. pylori eradication for primary prevention.

Our study had several limitations. First, we could not assess the gastric acid levels of individual patients. Secondly, this study was a retrospective study and the number of cases was small. Thirdly, it is possible that $H$. pylori-negative patients included not only those who were truly uninfected with $H$. pylori but also those whose $H$. pylori had been spontaneously eradicated, because $30 \%$ of the $H$. pylori-negative patients had opentype gastric atrophy. And there was some possibility of false positive in terms of current infection because all the patients with the positivity of $H$. pylori IgG antibody were diagnosed with $H$. pylori infection positive. Fourthly, we could not fully evaluate the influence of other drugs such as bisphosphonates, corticosteroids, and mucoprotective drugs. In the future, we plan to perform a multicenter, prospective study and screen for $H$. pylori infection in all subjects regardless of whether NSAIDs are used.

In conclusion, $H$. pylori exacerbates severe gastric mucosal injury in chronic NSAIDs users especially among the subjects of none or closed type gastric atrophy. Although the interaction between $H$. pylori and NSAIDs has not been fully elucidated, we hope that our data will be helpful for guiding the management and treatment of $H$. pylori-positive patients who use NSAIDs chronically. It is expected that $H$. pylori eradication would have a beneficial effect for the primary prevention of not only gastric ulcer and cancer, but also of severe gastric mucosal injury in chronic NSAIDs users.

\section{CONFLICTS OF INTEREST}

No potential conflict of interest relevant to this article was reported.

\section{ACKNOWLEDGEMENTS}

The sponsor had no role in the design of the study, data collection, analysis and interpretation, writing of the manuscript, or decision to submit for publication. The sponsor had no access to raw data. All authors had access to the raw data. The corresponding author had full access to all of the data and the final responsibility to submit for publication.

\section{REFERENCES}

1. Huang JQ, Sridhar S, Hunt RH. Role of Helicobacter pylori infection and non-steroidal anti-inflammatory drugs in peptic-ulcer disease: a meta-analysis. Lancet 2002;359:14-22.

2. Nishikawa K, Sugiyama T, Kato M, et al. Non-Helicobacter pylori and non-NSAID peptic ulcer disease in the Japanese population. Eur J Gastroenterol Hepatol 2000;12:635-640.

3. Lanza FL, Chan FK, Quigley EM; Practice Parameters Committee of the American College of Gastroenterology. Guidelines for prevention of NSAID-related ulcer complications. Am J Gastroenterol 2009;104:728-738.

4. Malfertheiner P, Chan FK, McColl KE. Peptic ulcer disease. Lancet 2009;374:1449-1461

5. Elliott SL, Ferris RJ, Giraud AS, Cook GA, Skeljo MV, Yeomans ND. Indomethacin damage to rat gastric mucosa is markedly dependent on luminal pH. Clin Exp Pharmacol Physiol 1996;23:432-434.

6. Nishino M, Sugimoto M, Kodaira C, et al. Relationship between low-dose aspirin-induced gastric mucosal injury and intragastric pH in healthy volunteers. Dig Dis Sci 2010;55:1627-1636.

7. McColl KE, el-Omar E, Gillen D. Helicobacter pylori gastritis and gastric physiology. Gastroenterol Clin North Am 2000;29:687703, viii.

8. Iijima K, Ara N, Abe Y, et al. Biphasic effects of H. pylori infection on low-dose aspirin-induced gastropathy depending on the gastric acid secretion level. J Gastroenterol 2012;47:1290-1297.

9. Fukuzawa M, Kawai T, Watanabe M, Tomiyama H, Yamashina A, Moriyasu F. Correlation between Helicobacter pylori infection and low-dose aspirin use on damage of the upper gastrointestinal tract. J Gastroenterol Hepatol 2012;27 Suppl 3:76-81.

10. Venerito M, Malfertheiner P. Interaction of Helicobacter pylori infection and nonsteroidal anti-inflammatory drugs in gastric and duodenal ulcers. Helicobacter 2010;15:239-250.

11. Lanza FL, Graham DY, Davis RE, Rack MF. Endoscopic comparison of cimetidine and sucralfate for prevention of naproxeninduced acute gastroduodenal injury: effect of scoring method. Dig Dis Sci 1990;35:1494-1499.

12. Kimura K, Takemoto T. An endoscopic recognition of the atrophic 
border and its significance in chronic gastritis. Endoscopy 1969;1: 87-97.

13. Iijima K, Ara N, Abe Y, et al. Association of gastric acid and mucus secretion level with low-dose aspirin-induced gastropathy. J Gastroenterol 2012;47:150-158.

14. Dixon MF, Genta RM, Yardley JH, Correa P. Classification and grading of gastritis: the updated Sydney System. International Workshop on the Histopathology of Gastritis, Houston 1994. Am J Surg Pathol 1996;20:1161-1181.

15. Sasazuki S, Inoue M, Iwasaki M, et al. Effect of Helicobacter pylori infection combined with CagA and pepsinogen status on gastric cancer development among Japanese men and women: a nested case-control study. Cancer Epidemiol Biomarkers Prev 2006;15: 1341-1347.

16. Yamamoto T, Ishii T, Sanaka M, Kuyama Y. Diagnosis of Helicobacter pylori infection using RAPIRUN H. pylori antibody detection kit. Expert Rev Mol Diagn 2008;8:565-569.

17. Kubota K, Shimoyama S, Shimizu N, et al. Studies of 13C-urea breath test for diagnosis of Helicobacter pylori infection in patients after partial gastrectomy. Digestion 2002;65:82-86.

18. Chan FK. Helicobacter pylori, NSAIDs and gastrointestinal haemorrhage. Eur J Gastroenterol Hepatol 2002;14:1-3.

19. Iijima K, Ara N, Abe Y, et al. Gastric acid secretion level modulates the association between Helicobacter pylori infection and lowdose aspirin-induced gastropathy. J Gastroenterol 2011;46:612619.

20. Katelaris PH, Seow F, Lin BP, Napoli J, Ngu MC, Jones DB. Effect of age, Helicobacter pylori infection, and gastritis with atrophy on serum gastrin and gastric acid secretion in healthy men. Gut 1993; 34:1032-1037.

21. Papatheodoridis GV, Sougioultzis S, Archimandritis AJ. Effects of Helicobacter pylori and nonsteroidal anti-inflammatory drugs on peptic ulcer disease: a systematic review. Clin Gastroenterol Hepatol 2006;4:130-142.

22. Konturek SJ, Konturek PC, Brzozowski T, Konturek JW, Pawlik WW. From nerves and hormones to bacteria in the stomach: Nobel prize for achievements in gastrology during last century. J Physiol Pharmacol 2005;56:507-530.

23. Alderman BM, Ulaganathan M, Judd LM, et al. Insights into the mechanisms of gastric adaptation to aspirin-induced injury: a role for regenerating protein but not trefoil peptides. Lab Invest 2003;
83:1415-1425.

24. Konturek JW, Dembinski A, Konturek SJ, Stachura J, Domschke W. Infection of Helicobacter pylori in gastric adaptation to continued administration of aspirin in humans. Gastroenterology 1998;114: 245-255.

25. Laine L, Kivitz AJ, Bello AE, Grahn AY, Schiff MH, Taha AS. Double-blind randomized trials of single-tablet ibuprofen/highdose famotidine vs. ibuprofen alone for reduction of gastric and duodenal ulcers. Am J Gastroenterol 2012;107:379-386.

26. Nema H, Kato M. Comparative study of therapeutic effects of PPI and H2RA on ulcers during continuous aspirin therapy. World J Gastroenterol 2010;16:5342-5346.

27. Miyake K, Ueki N, Suzuki K, et al. Preventive therapy for non-steroidal anti-inflammatory drug-induced ulcers in Japanese patients with rheumatoid arthritis: the current situation and a prospective controlled-study of the preventive effects of lansoprazole or famotidine. Aliment Pharmacol Ther 2005;21 Suppl 2:67-72.

28. Bianchi Porro G, Parente F, Imbesi V, Montrone F, Caruso I. Role of Helicobacter pylori in ulcer healing and recurrence of gastric and duodenal ulcers in longterm NSAID users: response to omeprazole dual therapy. Gut 1996;39:22-26.

29. Hawkey CJ, Tulassay Z, Szczepanski L, et al. Randomised controlled trial of Helicobacter pylori eradication in patients on nonsteroidal anti-inflammatory drugs: HELP NSAIDs study. Helicobacter Eradication for Lesion Prevention. Lancet 1998;352:10161021.

30. Chan FK, Chung SC, Suen BY, et al. Preventing recurrent upper gastrointestinal bleeding in patients with Helicobacter pylori infection who are taking low-dose aspirin or naproxen. N Engl J Med 2001;344:967-973.

31. Pilotto A, Di Mario F, Franceschi M, et al. Pantoprazole versus one-week Helicobacter pylori eradication therapy for the prevention of acute NSAID-related gastroduodenal damage in elderly subjects. Aliment Pharmacol Ther 2000;14:1077-1082.

32. Lai KC, Lau CS, Ip WY, et al. Effect of treatment of Helicobacter pylori on the prevention of gastroduodenal ulcers in patients receiving long-term NSAIDs: a double-blind, placebo-controlled trial. Aliment Pharmacol Ther 2003;17:799-805.

33. Iijima K, Ohara S, Sekine H, et al. Changes in gastric acid secretion assayed by endoscopic gastrin test before and after Helicobacter pylori eradication. Gut 2000;46:20-26. 

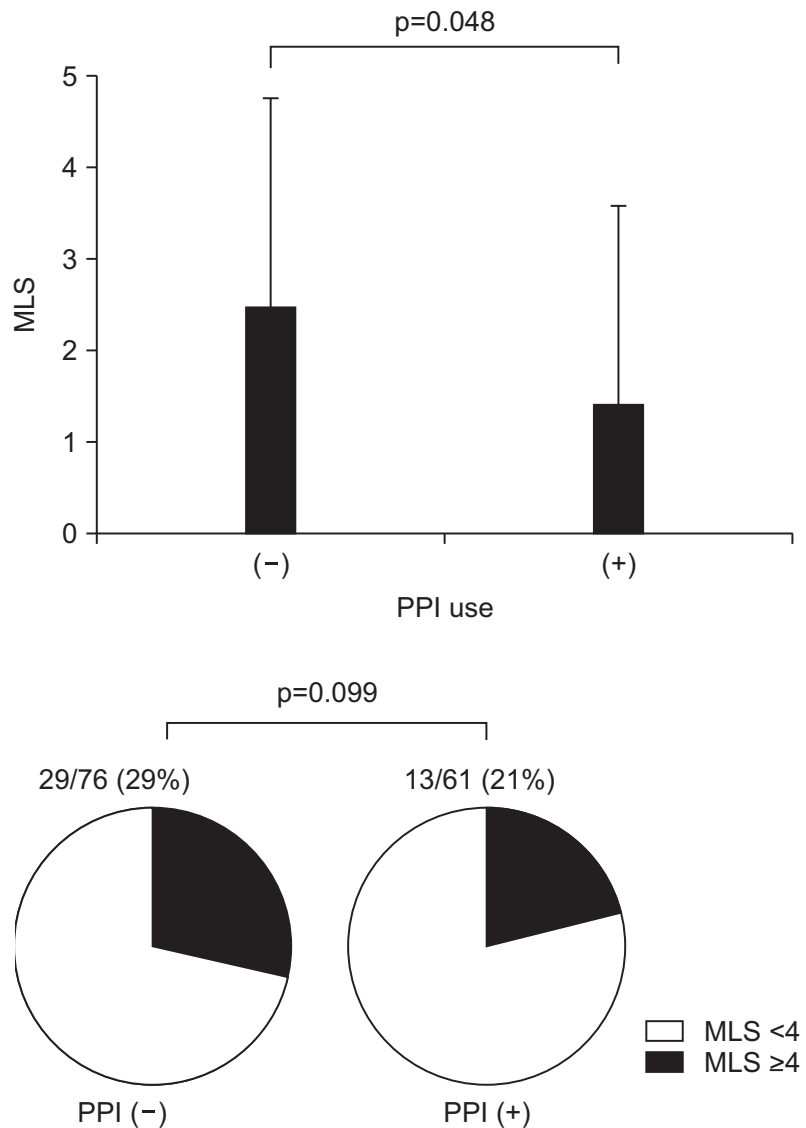

Supplementary Fig. 1. The degree of mucosal injury between proton pump inhibitor (PPI) users and PPI nonusers among Helicobacter pylori-positive subjects. The means of modified Lanza score (MLS) and the incidence of MLS $\geq 4$ were lower in the PPI users than in PPI nonusers among $H$. pylori-positive patients (each column indicates the mean $\pm \mathrm{SD} ; 2.5 \pm 2.3$ vs $1.5 \pm 2.2, \mathrm{p}=0.048$, Mann-Whitney U test; $29 \%$ vs $21 \%, p=0.099$, chi-square test). 